\title{
NOTE ON THE LAW OF BIQUADRATIC RECIPROCITY*
}

\author{
BY v. W. ADKISSON
}

In outlining the proof of the law of biquadratic reciprocity H. J. S. Smith develops the expressions for $S, T, S^{4}$, and $T^{4} \dagger$ which are used in the proof given by Eisenstein. $\ddagger$ We give here a slightly different development for these values making use of certain relationships established by Lebesgue. $\S$ The advantage in this development lies in the fact that the function $\psi(i)$ is exhibited in a form which shows it to be a polynomial in $i$ with integral coefficients, and that $\pm \psi(i)$ is a primary prime in the realm $k(i)$ if the proper sign be chosen. If

$$
F(\alpha)=\sum_{n=0}^{p-2} \alpha^{n} x^{g n},
$$

where $\alpha$ is a root of the equation $\left(\alpha^{p-1}-1\right) /(\alpha-1)=0, x$ is a root of $\left(x^{p}-1\right) /(x-1)=0, g$ is a primitive root of $p$, and $p$ is a prime of the form $4 n+1$, then

$$
F(\alpha) F\left(\alpha^{-1}\right)=\alpha^{(p-1) / 2} p .
$$

Substituting $i$ for $\alpha$, we obtain the result

$$
F(i) F(i)=F(-1) \sum_{t=1}^{p-2} i^{\text {ind } t}(-1)^{\operatorname{ind}(t+1)} \cdot \|
$$

Let

$$
\psi(i)=\frac{[F(i)]^{2}}{F(-1)}=\sum_{t=1}^{p-2} i^{\operatorname{ind} t}(-1)^{\operatorname{ind}(t+1)} .
$$

Hence, $\psi(i)$ is a polynomial in $i$ with integral coefficients, and may be written in the form $a+b i$ where $a$ and $b$ are integers. But

* Research paper No. 280, University of Arkansas.

$\dagger$ H. J. S. Smith, Collected Mathematical Papers, vol. 1, pp. 78-87.

$\ddagger$ E. Eisenstein, Lois de réciprocité, Journal für Mathematik (Crelle), vol. 28 , pp. $57-67$.

$\S$ L. M. V.-A. Lebesgue, Démonstration de quelques formules d'un mémoire de M. Jacobi, Journal de Mathématiques (Liouville), vol. 19, pp. 289.

|| Lebesgue, loc. cit. 


$$
\psi(i) \psi(-i)=\frac{[F(i) F(-i)]^{2}}{[F(-1)]^{2}}=\frac{\left[i^{(p-1) / 2} p\right]^{2}}{(-1)^{(p-1) / 2} p}=p .
$$

Therefore, $\psi(i)$ and $\psi(-i)$ are primes in $k(i)$. Since there are an even number of terms in (3) for which ind $t$ is odd, and an odd number of terms for which ind $t$ is even, $\pm \psi(i)$ and $\pm \psi(-i)$ will be primary primes, $a+b i$ and $a-b i$ respectively, in $k(i)$ if the proper signs be taken in each case. Moreover, $\psi(-i)$ [or $-\omega(-i)]$ is primary if $\psi(i)[$ or $-\psi(i)]$ is primary, and hence $(a+b i)(a-b i)=p$.

Since $g$ is a primitive root of $p$, we have

$$
g^{(p-1) / 2} \equiv-1, \bmod p ; \quad g^{(p-1) / 4} \equiv i, \quad \bmod p_{1} ;
$$

and

$$
g^{(p-1) / 4} \equiv-i, \bmod p_{2},
$$

where $p_{1} p_{2}=p$. Then $\left(g / p_{1}\right)_{4}=i$.

Let $g^{s} \equiv k \bmod p$; then since

we find

$$
F(i)=\sum_{s=0}^{p-2} i^{s} x^{g^{s}}
$$

$$
F(i)=\sum_{s=0}^{p-2}\left(\frac{g^{s}}{p_{1}}\right)_{4} x^{g^{s}}=\sum_{k=1}^{p-1}\left(\frac{k}{p_{1}}\right)_{4} x^{k}=S .
$$

From (3) and (4),

$$
[F(i)]^{4}=[F(-1)]^{2}[\psi(i)]^{2}=p(a+b i)^{2}=p p_{1}^{2}=S^{4} .
$$

In like manner

and

$$
F(-i)=\sum_{k=1}^{p-1}\left(\frac{k}{p_{1}}\right)_{4}^{3} x^{k}=T
$$

$$
T^{4}=p(a-b i)^{2}=p p_{2}^{2} .
$$

University of Arkansas

* The symbol $\left(g / p_{1}\right)_{4}$ due to H. J. S. Smith is the power of $i$ which is congruent to $g^{(p-1) / 4} \bmod p_{1}$. 